\title{
Therapie von nervenbezogenen muskuloskelettalen Schmerzen
}

\author{
Colette Ridehalgh
}

\author{
Neurodynamische Behandlung? Welche Wirkung haben physiotherapeutische Techniken? \\ Auf welchen Mechanismen beruhen sie? Der Artikel gibt Antworten auf diese Fragen anhand \\ der aktuellen Evidenzlage. Klar ist: Es liegt viel Potential in der Neurodynamik.
}

Es gibt keine „Zauberformel“ für die Behandlung von Menschen mit nervenbezogenen muskuloskelettalen Schmerzen. Die Ergebnisse des Assessments bestimmen daher, welcher Patient welche Behandlung erhält. In erster Linie (vorausgesetzt, es bestehen keine Red Flags), werden dabei im Wesentlichen zwei Ziele verfolgt: die Wiederherstellung der Nervenfunktion und/oder die Reduktion erhöhter neuraler Mechanosensitivität. Es kann jedoch sein, dass man beide Probleme nicht gleichzeitig angeht, denn es ist möglich, dass bei einem Patienten neurologische Integritätstests (Kraft, Sensibilität, Reflexe) positiv und gleichzeitig neurodynamische Tests negativ ausfallen und umgekehrt. In einer Studie von Baselgia et al. zeigten mehr als 54\% der Patienten mit Karpaltunnelsyndrom zwar normale neurodynamische Tests, aber eine Einschränkung bei der Temperaturempfindung [1], was bedeutet, dass ein Verlust der Nervenfunktion und eine erhöhte neurale Mechanosensitivität nicht immer gemeinsam auftreten.

Zu den Optionen für primär auf Nerven fokussierte Behandlungstechniken gehören mechanische InterfaceTechniken, Techniken zur Nervenmobilisation (Nerve Mobilisation Techniques (NMT), z. B. Tensioner- oder SliderTechniken) oder eine Kombination aus beiden. In den folgenden Abschnitten werden diese Techniken, ihre postulierten Wirkungen und die aktuelle Evidenzlage beschrieben.

\section{Mechanische Interface-Techniken}

Nerven verlaufen entlang der Extremitäten und des Rumpfes durch und in der Nähe von vielen unterschiedlichen Geweben. Diese Gewebe werden als mechanisches Interface bezeichnet, da die Nerven bei Bewegungen der Extremitäten und des Rumpfes in Relation zu diesen Geweben gleiten. Die Gefahr eines Entrapments oder einer Irritation des Nervs besteht gerade aufgrund dieses engen Zusammenhangs. Die häufigsten Interface-Dysfunktionen treten am Karpaltunnel, den N. medianus betreffend, und am Spinalkanal oder Foramen intervertebrale, die Nervenwurzeln betreffend, auf. In diesen Fällen könnte die Behandlung der Gewebe und Strukturen um den Nerv die Durchblutung erhöhen, die Auflösung von Ödemen unterstützen, Schmerzen durch absteigende inhibitorische Schmerzmechanismen lindern und die umgebenden Gewebe mobilisieren, was zu einer Verbesserung der Nervenexkursion durch diese Gewebe führen könnte [2]. Alle diese Effekte können dazu beitragen, die Nervenfunktion zu verbessern oder die erhöhte neurale Mechanosensitivität zu reduzieren. Es gibt jedoch nur relativ wenig eindeutige Evidenz für die Wirksamkeit dieser Mechanismen.

\section{Evidenz der Interface-Techniken}

Während die bei Interface-Techniken wirksamen Mechanismen zu wenig erforscht sind, liefern einige Wirksamkeitsstudien vielversprechende Ergebnisse. In einer Studie von Tal-Akabi und Rushton schnitten Mobilisationen der Handwurzelknochen bei Karpaltunnelsyndrom positiver als NMT und signifikant besser als eine Kontrollgruppe ab [3]. Eine solche karpale Mobilisation kann insofern als Interface-Technik betrachtet werden, als dass sie das Potential hat, auf den Karpaltunnel selbst einzuwirken. Möglicherweise spielen Mechanismen der absteigenden Schmerzinhibition, die für viele positive Behandlungsergebnisse verantwortlich sind, auch hier eine Rolle [4][5] [6]. Rodriguez-Sanz et al. stellten außerdem fest, dass bei Patienten mit zervikobrachialen Schmerzen eine kontralaterale Side-Glide-Technik ( $\triangleright$ Abb. 1) einer Kontrollgruppe, die auf einer Warteliste stand, überlegen war und eine statistisch signifikante Reduzierung der Schmerzintensität anhand einer Numerischen Rating Scale zeigte [7].

\section{Neurodynamische Techniken (NMT)}

Direktere Formen der Nervenmobilisation, bei denen neurodynamische Techniken zum Einsatz kommen, wurden während der letzten Jahre einer genaueren Untersuchung unterzogen. Sie werden häufig in Sliders und Tensioners unterteilt [8]. 

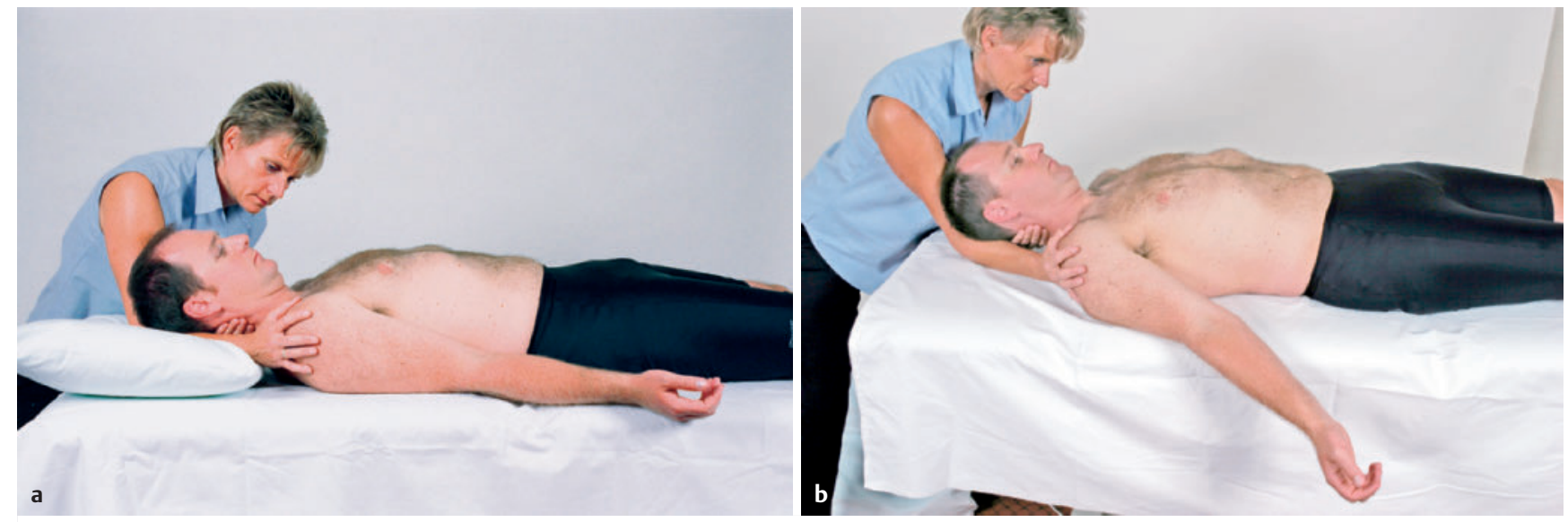

- Abb. 1 Side Glide Techniken an der HWS [9]. a Side Glide Technik an der HWS [9]. b Side Glide Technik an der HWS unter vermehrter neurodynamischer Belastung [9]. (Quelle: van den Berg F 2016, Hrsg. Physiotherapie für alle Körpersysteme. 1. Auflage. Stuttgart: Thieme; 2016. doi:10.1055/b-004-129718)
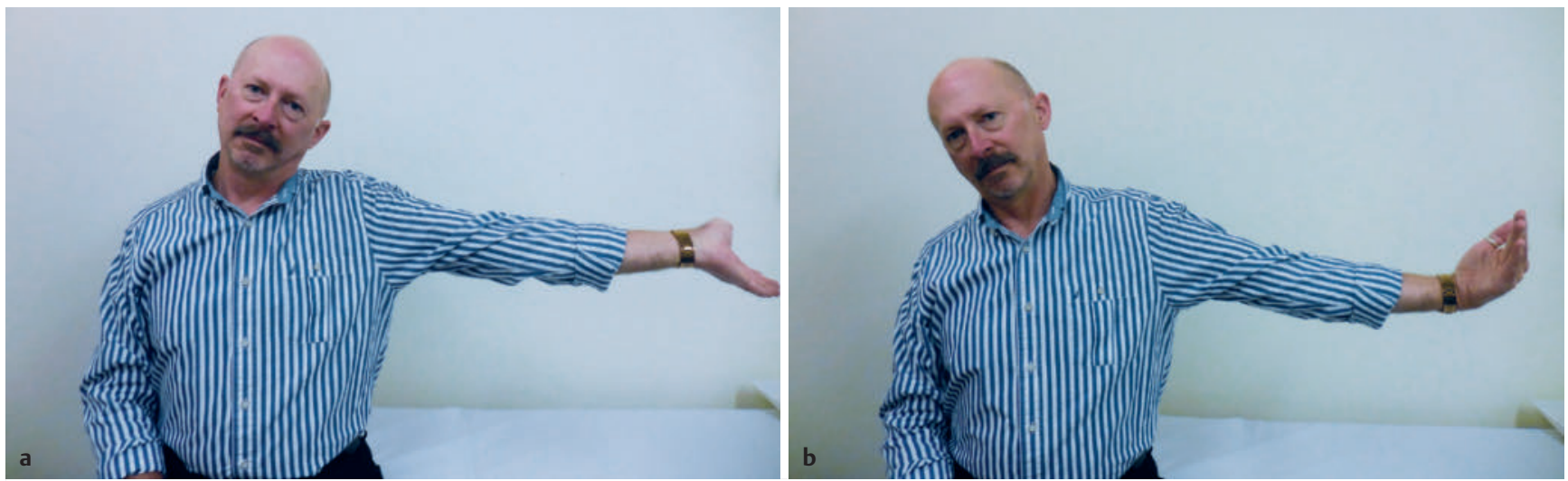

- Abb.2 Slider für den Arm (Tendenz N. medianus) (Symbolbilder). a Slider für den Arm (Tendenz N. medianus): Halswirbelsäule wird ipsilateral zur Seite flektiert, während das Handgelenk extendiert wird. b Slider für den Arm (Tendenz N. medianus): Halswirbelsäule wird kontralateral zur Seite flektiert, während das Handgelenk flektiert wird, um eine größere Exkursion zu bewirken. (Quelle: B. Tampin)

Slider Bei Slider-Techniken werden die Gelenke an einem Ende einer Extremität so positioniert, dass das Nervenbett verlängert wird, während die Gelenke am anderen Ende so eingestellt werden, dass sie eine Verkürzung des Nervenbetts bewirken ( $\triangleright$ Abb. 2 ).

Tensioner Bei einem Tensioner werden beide Enden der neurodynamischen Position zusammen eingestellt, sodass insgesamt ein Verlängerungseffekt auftritt ( A Abb. 3).

Der Grundgedanke ist, dass bei einem Slider eine größere Exkursion und weniger Spannung, bei einem Tensioner hingegen mehr Spannung und weniger Exkursion auftritt. Dies wurde in Kadaverstudien nachgewiesen [8] und auch durch Ultraschalluntersuchungen in vivo gestützt [10][11] [12]. Wie dem auch sei, wird jedoch nicht in den Endbereichen des Bewegungsumfangs behandelt, ist es in der klinischen Praxis nicht möglich, das Verhältnis von Spannung zu Exkursion zu quantifizieren. In bestimmten Positionen kann ein Tensioner sogar weniger Spannung bewirken als ein Slider [11]. Bei einem modifizierten Straight Leg Raise $(S L R)$ in Seitenlage z. B. führte die Flexion der Hüfte auf $60^{\circ}$ zu einer größeren Exkursion des Ischiasnervs als die Flexion auf $30^{\circ}$ ( $\triangleright$ Abb.4) [13]. Wäre die Hüftflexion jedoch bis in die Endbereiche des Bewegungsumfangs erweitert worden, hätte die Exkursion wahrscheinlich ab- und die Spannung zugenommen.
Bei der Behandlung muss
berücksichtigt werden, dass
nicht überprüft werden kann, was biomechanisch passiert. Schweregrad, Irritierbarkeit und Symptome, die während der Tests hervorgerufen werden, sind daher von essenzieller Bedeutung für die Wahl der Behandlungsmethoden. 


\section{Wirkungen der Techniken}

Während es bisher wenig Forschung zur Wirksamkeit von Interface-Techniken gibt, sind die Wirkungen von NMT Gegenstand zahlreicher Untersuchungen und Artikel. Zu diesen Wirkungen gehören Linderung von intraneuralen Ödemen, hypoalgetische Effekte, Veränderung der Immunzellenaktivierung, Nervenregeneration und biomechanische Effekte [14][15][16][17][18][19][20].

\section{Linderung von Ödemen}

Intraneurale Ödeme können den Druck auf den Nerv erhöhen und eine Entzündungssuppe enthalten, die den Nerv ebenfalls sensibilisieren kann [21]. Daher kann die Auflösung von Ödemen viele positive Auswirkungen auf den Gesundheitszustand des Patienten haben. In drei Kadaverstudien wurde gezeigt, dass wiederholte Bewegungen einen Farbstoff verteilen können, der in den N. tibialis [14], die Nervenwurzel [15] oder den N. medianus [16] injiziert wurde. Interessanterweise war es für das Auftreten dieses Effekts unerheblich, ob ein Slider oder ein Tensioner eingesetzt wurde [16]. Möglich ist jedoch, dass sich diese Vorgänge in vivo nicht auf genau dieselbe Weise abspielen.

In vivo Evidenz zur Auflösung von Ödemen findet sich bei Schmid et al. [17]. Slider-Mobilisationen, die über eine Woche jeden Tag angewandt wurden, führten zu einer signifikanten Reduzierung der MRI Ratio Signal Intensity (Zeichen für ein Ödem) proximal des Karpaltunnels. Das Tragen von Schienen in der Nacht führte zu vergleichbaren Resultaten, was bedeutet, dass die Kombination aus täglichen NMT und nächtlicher Schienung gut geeignet ist, um die Auflösung von Ödemen voranzutreiben. Ob Schmerzen sich aufgrund der Auflösung von Ödemen ändern, ist unklar, aber eine Reihe von Tierstudien zeigte, dass NMT bei einem bestimmten Nervenverletzungsmodell das Schmerzverhalten von Ratten verbessern können [18][19][20].

\section{Verbesserung von Schmerzen}

Bertolini et al. und Santos et al. verwendeten SLR-Tensioners und zeigten, dass selbst bei Tieren mit schweren Nervenverletzungen eine Schmerzlinderung bewirkt werden kann [18][20]. Tensioners werden oft als die aggressivere Technik betrachtet, aber diese Studien zeigten, dass sie hypoalgetisch wirken können. Außerdem beobachteten Martins et al. [19] und Santos et al. [20] nicht nur eine Schmerzlinderung, sondern auch eine Verbesserung der Immunzellenaktivierung (Gliazellen).

\section{Tensioners werden zwar oft als die aggressivere Technik gegenüber Sliders gesehen, können aber auch hypoalgetisch wirken.}

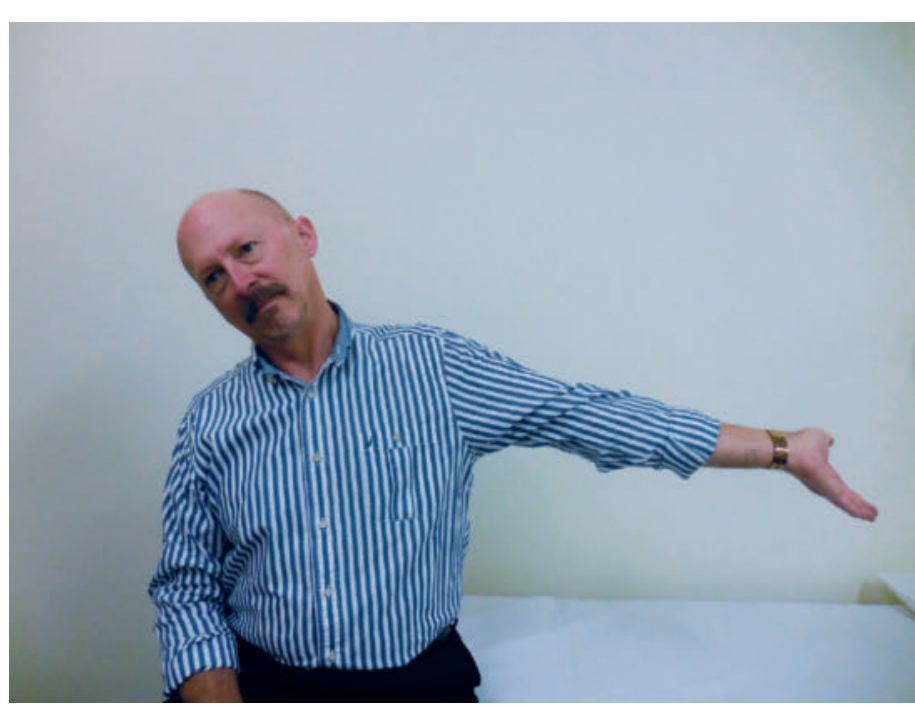

- Abb.3 Tensioner für den Arm (Tendenz N. medianus). Halswirbelsäule und Handgelenk werden so positioniert, dass der Nerv (peripherer Nerv und Nervenwurzeln) eine längere Strecke zurücklegen muss, was zu mehr Spannung und weniger Exkursion führt (Symbolbild). (Quelle: B. Tampin)

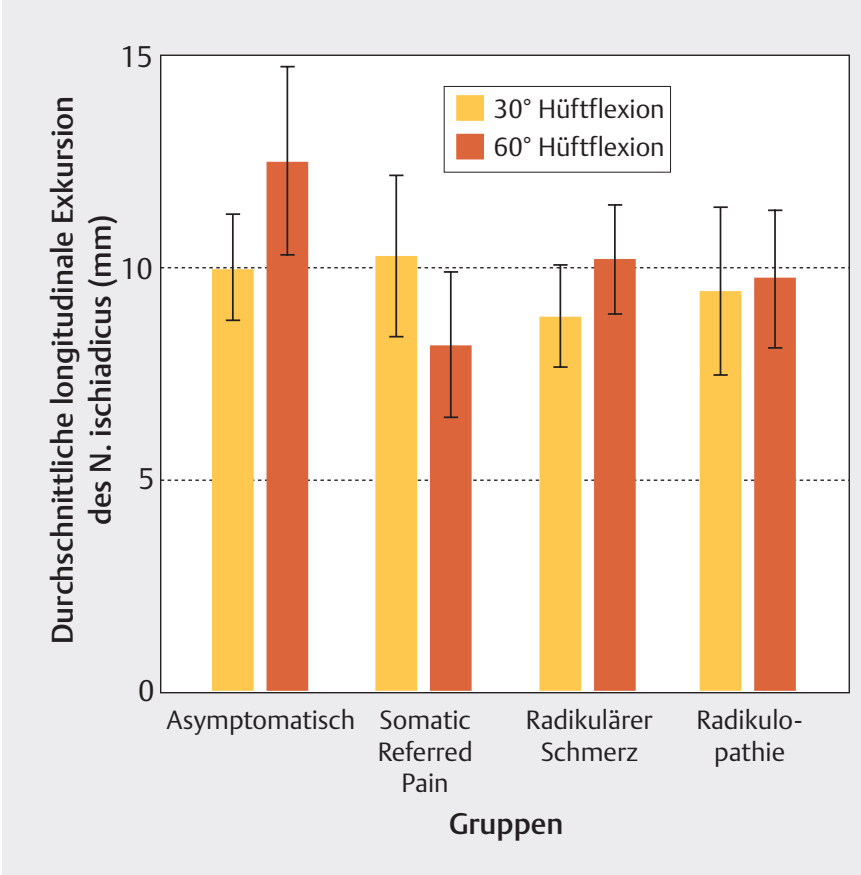

Abb. 4 Exkursion des N. ischiadicus in Millimeter, gemessen mit Ultraschall im posterioren Oberschenkel bei einem modifizierten SLR-Test mit $30^{\circ}$ und $60^{\circ}$ Hüftflexion. Nur bei der Gruppe „Somatic Referred Pain“ war bei größerer Hüftflexion eine Abnahme der Exkursion zu beobachten, bei allen anderen Gruppen nahm die Exkursion zu (Ridehalgh et al. 2015) [13]. (Quelle: C. Ridehalgh; graf. Umsetzung: Thieme Gruppe)

\section{Verbesserung der Immunzellenenaktivierung}

Nach einer Nervenverletzung werden Gliazellen im Rückenmark aktiviert [22][23], was zu einer Produktion und Ausschüttung von proinflammatorischen Mediatoren wie Zytokinen, Prostaglandinen und neurotrophen Faktoren 
führt. Bei Experimenten mit Ratten stieg die Gliazellenaktivierung im Hinterwurzelganglion und im Hinterhorn nach einer Nervenverletzung an und normalisierte sich nach Mobilisation wieder [19][20]. In jüngeren Studien wurden vergleichbare Veränderungen sowohl im periaquäduktalen Grau im Mittelhirn als auch im Thalamus beobachtet, was auf Veränderungen von Immunzellen in einem großen Teil des Nervensystems nach neuralen Mobilisationen hindeutet [24]. Nach aerobem Training wurden zwar Veränderungen der Immunzellen im Hinterhorn beobachtet, jedoch nicht im Gehirn [25], was darauf schließen lässt, dass neurale Mobilisationen einzigartige Wirkungen haben, die bei gewöhnlichen Übungen allein nicht auftreten.

\section{Regeneration von Nervenfasern}

Ein weiterer wichtiger Effekt, der in Tierstudien nachgewiesen wurde, ist die Regeneration von sowohl myelinisierten als auch unmyelinisierten Fasern nach neuraler Mobilisation [26]. Möglicherweise setzen diese Techniken also einen Reparaturprozess in Gang.

\section{Limitation}

Diese innovativen Studien liefern einerseits starke Evidenz für die Mechanismen, die nach NMT auftreten, sind anderseits jedoch in ihrer Aussagekraft eingeschränkt, da es sich ausschließlich um Studien mit Nagetieren handelt. Unklar ist, ob dieselben Effekte in demselben Maß bei Menschen auftreten würden. Zurzeit ist es nicht möglich, diese Mechanismen in vivo bei Menschen zu untersuchen, allerdings wurden biomechanische Effekte erforscht.

\section{Veränderung der Biomechanik}

In der Fachliteratur wird darüber diskutiert, ob nach einer Nervenverletzung biomechanische Veränderungen auftreten. In einer Ultraschallstudie konnten in Bezug auf die Exkursion des Ischiasnervs keine Unterschiede zwischen asymptomatischen Teilnehmern und Personen mit Beinschmerzen, die von der Wirbelsäule ausstrahlen (Spinally Referred Leg Pain - SRLP), festgestellt werden [13]. Aufgrund der großen Nähe zu den Wirbeln ist es jedoch nicht möglich, die lumbosakralen Nervenwurzeln per Ultraschall zu beurteilen. Da es daher nicht möglich war, die Exkursion näher an der Nervenläsion zu scannen, könnte es also sein, dass mögliche Veränderungen nicht sichtbar waren. Im Gegensatz dazu kommt ein aktuelles systematisches Review von Ellis et al. zu dem Ergebnis, dass die Exkursion des N. medianus bei Patienten mit Karpaltunnelsyndrom im Vergleich zu gesunden Studienteilnehmern reduziert ist [27]. Noch aktueller ist die Hypothese, dass Nerven nach einer Verletzung steifer werden [28]. Allerdings steckt die Technologie, die bei entsprechenden Untersuchungen zum Einsatz kam (Scherwellen-Elastografie), noch in den Kinderschuhen und ihre Validität muss erst noch nachgewiesen werden.
In Bezug auf Veränderungen der Biomechanik bei Patienten mit SRLP gibt es eine Studie, in der nach drei einminütigen SLR-Tensioner-Mobilisationen keinerlei Veränderung der Exkursion des Ischiasnervs beobachtet wurde [29]. Zwar ist die Studie dadurch eingeschränkt, dass der Ischiasnerv und nicht die Nervenwurzel gescannt wurde, aber dennoch könnte man erwarten, dass aufgrund der Kontinuität des Nerventrakts jede Verbesserung der Exkursion auch distal der behandelten Region auftreten sollte. Neto et al. konnten keine Veränderung der Steifheit des Ischiasnervs nach einer gehaltenen Dehnung in der SLUMP-Position feststellen [30]. Da in dieser Studie jedoch junge, asymptomatische Teilnehmer involviert waren, lässt sich nicht sagen, ob bei symptomatischen Personen dieselben Resultate auftreten würden.

Zusätzlich zur Anwendung von isolierten Tensioners oder Sliders besteht auch die Möglichkeit, eine Interface-Technik mit einem Tensioner oder Slider zu kombinieren. Der Vorteil dieser Kombination ist, dass der größte Einfluss auf das Nervengewebe an einem bestimmten Punkt innerhalb des Bewegungsumfangs stattfinden könnte. Beispielsweise stellten Kobayashi et al. fest, dass bei Patienten mit lumbosakraler Bandscheibenhernie die Einschränkung ihres SLR mit dem Bewegungsumfang des SLR übereinstimmte, der während einer Operation auftrat, also in einer Situation, wenn die Nervenwurzel und die Durchblutung am stärksten eingeschränkt waren [31]. Daher kann es sein, dass eine Interface-Technik eine größere Wirkung (biomechanisch als auch neurophysiologisch) erzielt, wenn sich der Körper in der provokativen neurodynamischen Position befindet.

\section{Wirksamkeit von neurodynamischen Techniken}

Zwar gibt es Evidenz zugunsten der Mechanismen von neuralen Interventionen, aber es ist wichtig zu wissen, ob diese Techniken auch die Outcomes der Patienten mit nervenbezogenen Schmerzen verbessern. Während der letzten Jahre sind entsprechende Wirksamkeitsstudien zu widersprüchlichen Ergebnissen gekommen. Wichtige Faktoren für die unterschiedlichen Resultate waren die behandelte Körperregion, die betroffenen Nerven, die verschiedenen Populationen und die Wahl der Techniken. In dem Versuch, die Fachliteratur zur Wirksamkeit zusammenzufassen, bezieht sich der folgende Abschnitt auf einige in letzter Zeit veröffentlichten systematischen Reviews.

In zwei aktuellen systematischen Reviews wird die Literatur zu NMT in vielerlei Hinsicht kritisiert [32][33]. Su et al. beurteilten in ihrem Review die Wirksamkeit der NMT versus minimalen oder anderen Interventionen [33], während Basson et al. in ihrem Review die NMT anhand verschiedener Krankheitsbilder bewerteten [32]. Verglichen mit minimaler Intervention erwies sich NMT 
als überlegen in Bezug auf Schmerzlinderung und Behinderung, aber die gepoolten Resultate sprachen nicht für eine Überlegenheit von NMT gegenüber anderen Interventionen und es wurden nur kurzfristige Ergebnisse bewertet (maximal zehn Wochen nach der Behandlung) [33]. Basson et al. bescheinigten NMT eine Wirksamkeit in Bezug auf eine kurzfristige Schmerzlinderung und Verbesserung der Behinderung bei Patienten mit SRLP (bis zu acht Wochen nach der Behandlung) und in Bezug auf die Schmerzlinderung bei Patienten mit Nacken-ArmSchmerzen [32]. Bei Patienten mit Karpaltunnelsyndrom erwies sich NMT nicht als effektiv, obwohl hier Hinweise auf positive neurophysiologische Effekte vorlagen. Es ist daher möglich, dass die Resultate von Su et al. darauf zurückzuführen sind, dass in ihrem Review Studien zusammengefasst wurden, die sich auf Schmerzen in unterschiedlichen Körperregionen beziehen [33]. Hinzu kommt, dass die Zahl der Studien in der Kategorie „andere Interventionen“ vergleichsweise gering war.

\section{Patienten mit Kreuzschmerzen}

Neto et al. konzentrierten ihr systematisches Review auf die Wirksamkeit von NMT im unteren Quadranten bei gesunden Studienteilnehmern und bei Patienten mit Kreuzschmerzen [34]. Ihre Resultate legen den Schluss nahe, dass NMT bei gesunden Personen die Flexibilität der Beine verbessern kann. Diese Verbesserungen des Bewegungsumfangs können jedoch auch auf eine Verlängerung der Muskeln und somit nicht direkt auf Veränderungen der Nerven zurückzuführen sein. Im Hinblick auf Studienteilnehmer mit Kreuzschmerzen stützen Neto et al. die Ergebnisse von Basson et al. [32] insofern, als dass sie ebenfalls signifikante Verbesserungen in Bezug auf Schmerzen und Behinderung nach NMT konstatierten. Diese Übereinstimmung mag einerseits nicht überraschen, da beide Reviews im selben Jahr veröffentlicht wurden, aber andererseits überschneiden sich die Reviews nur in Bezug auf zwei Studien.

\section{Patienten mit Karpaltunnelsyndrom}

Was Patienten mit Karpaltunnelsyndrom betrifft, kamen Ballestero-Pérez et al. in einem Review zu gemischten Ergebnissen in Bezug auf Schmerzen und Funktion [35]. Positiv ist, dass in zwei der ausgewerteten Studien die Mehrheit der Teilnehmer, die NMT erhielten, nicht operiert werden musste [3][36]. Allerdings kann nur die Hälfte der in den Review einbezogenen Studien in Hinblick auf ihren PEDro-Wert als gut eingestuft werden. Daher müssen die Resultate des Reviews angesichts der Bias und Qualität eines Großteils der Studien kritisch gesehen werden. Es sollte auch berücksichtigt werden, dass die Sehnen- und Nervengleittechniken, die in einigen Studien der Reviews von Ballestero-Pérez et al. [35] und Basson et al. [32] angewandt wurden, sich von NMT unterscheiden, da sie auf Bewegungen in der Hand beschränkt sind. Möglicherweise sind die Wirkungen lokalisierter Bewegungen weniger signifikant als Bewegungen, bei denen der Nerv über die gesamte Extremität entlang des Nervenbetts bewegt wird.

\section{Subgruppenbildung}

Auch wenn sie mit vielen Einschränkungen behaftet sind, gelten systematische Reviews, und vor allem solche, die eine Meta-Analyse enthalten, als Goldstandard innerhalb der Evidenzhierarchie [37]. Eine der wichtigsten Einschränkungen ist, dass Patienten, die einen Physiotherapeuten aufsuchen, nicht zufällig in die eine oder andere Behandlungsgruppe eingeteilt werden. Sie werden nach einem ganzheitlichen, personenzentrierten Ansatz untersucht und behandelt. Im Rahmen einer randomisierten kontrollierten Untersuchung kann dies problematisch sein, da Variablen streng kontrolliert werden müssen, aber zumindest sollte der Versuch unternommen werden, eine angemessene Einteilung in eine Subgruppe zu gewährleisten. Es konnte nachgewiesen werden, dass bestimmte Subgruppen bei Patienten mit nervenbezogenen Schmerzen existieren und dass jede Gruppe unterschiedlich auf bestimmte Behandlungsmethoden reagieren kann [38][39]. Schäfer et al. stellten fest, dass Studienteilnehmer mit rückenbedingten Beinschmerzen und erhöhter neuraler Mechanosensitivität nach neuraler Mobilisation signifikant größere Verbesserungen in global wahrgenommener Veränderung zeigten als Patienten in anderen Subgruppen [39]. Die Teilnehmerzahl in der Gruppe „erhöhte neurale Mechanosensitivität“ war klein $(n=9)$, was die Extrapolation auf die Praxis einschränkt, aber diese Resultate zeigen, dass die Existenz von Subgruppen intensiver erforscht werden muss, damit Patienten danach klassifiziert werden können, welche Behandlungsmethode für sie am besten geeignet ist.

\section{Um die bestmögliche Therapie anbieten zu können, sollte die Existenz von Subgruppen bei Patienten mit nervenbezogenen Schmerzen intensiver erforscht werden}

\section{Sicherheit der neurodynamischen Mobilisation}

Ein wichtiger Faktor bei der Entscheidung für oder gegen NMT ist die Sicherheit des Patienten. Es wurden Befürchtungen geäußert, dass vor allem Tensioners die Blutversorgung von bereits beeinträchtigten Nerven verschlechtern könnten, was zu einem weiteren Verlust der Nervenfunktion [40] oder einer Verschlechterung des Schmerzverhaltens [14] führen könnte. Die bereits genannten Tierstudien zeigten jedoch, dass sich auch bei Anwendung von Tensioners zahlreiche Messwerte verbessern können. Hinzu kommt, dass selbst bei Patienten 
mit Radikulopathie die Anwendung von Tensioners keine negative Auswirkung auf die Vibrationswahrnehmungsschwelle (ein Messwert für die Funktion von A $\beta$-Nervenfasern) hatte [41]. Allerdings wurden in dieser Studie Patienten mit schweren und irritierbaren Symptomen ausgeschlossen [41]. Nee et al. kamen zu dem Schluss, dass sich bei Patienten mit Nacken-Arm-Schmerzen die Wahrscheinlichkeit einer Verbesserung durch NMT nicht verringerte, auch wenn die Patienten nach einer Behandlung zunächst eine Verschlechterung erlebten [42]. In dieser Studie wurden die NMT-Techniken allerdings entsprechend den Symptomen der Patienten intensiviert. Daher sollten, wie bei allen Behandlungsstrategien, auch bei NMT die Schwere der Erkrankung und die Irritierbarkeit des Patienten bei der Behandlungsplanung berücksichtigt werden.

\section{Fazit}

Neurodynamische Techniken (NMT) haben nachweislich verschiedene wichtige neurophysiologische und biomechanische Wirkungen. Sowohl Tensioner- als auch SliderTechniken können gefahrlos eingesetzt werden, wenn die Schwere der Erkrankung und die Irritierbarkeit des Patienten angemessen berücksichtigt werden. Studien zur Wirksamkeit sprechen für NMT, sind jedoch nicht eindeutig in Bezug auf Patienten mit Karpaltunnelsyndrom. Die Aussagekraft vieler randomisierter kontrollierter Studien ist auf die eine oder andere Weise eingeschränkt. Für zukünftige Studien sollten andere methodische Vorgehensweisen oder eine angemessene Subgruppenbildung in Betracht gezogen werden, um ein realistischeres Verständnis der Ergebnisse für Patienten mit nervenbezogener muskuloskelettaler Dysfunktion zu ermöglichen.
Autorinnen/Autoren

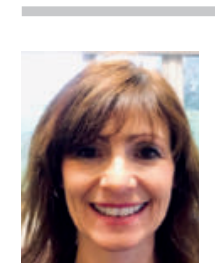

\section{Dr. Colette Ridehalgh}

ist Senior Physiotherapy Lecturer an der University of Brighton und unterrichtet Studierende (undergraduate \& postgraduate) in muskuloskelettaler Physiotherapie. Sie hat sowohl innerhalb des National Health Service (NHS) in England als auch in der Privatpraxis und an der Universität gearbeitet. Ihr besonderes Interesse gilt der Behandlung von Menschen mit nervenbezogenen muskuloskelettalen Schmerzen. Sie hat einen MSc in Manipulative Physiotherapy und ist Mitglied der Musculoskeletal Association of Chartered Physiotherapists. 2014 schloss sie ihre Promotion zu dem Thema Straight Leg Raise Behandlung für Menschen mit von der Wirbelsäule ausstrahlenden Beinschmerzen ab und veröffentlichte eine Reihe von Peer-Reviewed-Artikeln. Ihre Arbeit stellte sie auf nationalen und internationalen Konferenzen vor.

\section{Korrespondenzadresse}

\section{Dr. Colette Ridehalgh}

cr19@brighton.ac.uk

Literatur

Literaturverzeichnis am Ende der HTML-Version unter www. thieme-connect.de/products/manuelletherapie

Bibliografie

DOI https://doi.org/10.1055/a-1080-8056

manuelletherapie 2020; 24 : 15-20

(c) Georg Thieme Verlag KG Stuttgart · New York ISSN 1433-2671 